\title{
COPD and cancer mortality: the influence of statins
}

\author{
Y R B M van Gestel, ${ }^{1}$ S E Hoeks, ${ }^{1}$ D D Sin, ${ }^{2}$ V Hüzeir, ${ }^{3}$ H Stam, ${ }^{4}$ F W Mertens, ${ }^{4}$ R T van \\ Domburg, ${ }^{3} \mathrm{~J} J \mathrm{Bax},{ }^{5}$ D Poldermans ${ }^{1}$
}

- Additional data are published online only at http://thorax.bmj. com/content/vol64/issue11

${ }^{1}$ Department of Anaesthesiology, Erasmus Medical Centre, Rotterdam, The Netherlands; ${ }^{2}$ Department of Medicine, University of British Columbia and The James Hogg iCAPTURE Centre, St Paul's Hospital, Vancouver, Canada;

${ }^{3}$ Department of Cardiology, Erasmus Medical Centre, Rotterdam, The Netherlands; ${ }^{4}$ Department of Pulmonology, Erasmus Medical Centre Rotterdam, The Netherlands; ${ }^{5}$ Department of Cardiology, Leiden University Medical Centre, Leiden, The Netherlands

Correspondence to:

Professor D Poldermans,

Erasmus Medical Centre

Rotterdam, 's-Gravendijkwal

230, 3015 CE Rotterdam, The

Netherlands; d.poldermans@

erasmusmc.nl

Received 25 March 2009 Accepted 12 August 2009

Published Online First

27 August 2009

\section{ABSTRACT}

Background: Chronic obstructive pulmonary disease (COPD) is associated with an increased risk of lung cancer, independently of smoking. However, the relationship between COPD and total cancer mortality is less certain. A study was undertaken to investigate the association between COPD and total cancer mortality and to determine whether the use of statins, which have been associated with cancer risk in other settings, modified this relationship.

Methods: The study included 3371 patients with peripheral arterial disease who underwent vascular surgery between 1990 and 2006; 1310 (39\%) had COPD and the rest did not. The primary end point was cancer mortality (lung and extrapulmonary) over a median followup of 5 years.

Results: COPD was associated with an increased risk of both lung cancer mortality (hazard ratio (HR) 2.06; $95 \% \mathrm{Cl}$ 1.32 to 3.20 ) and extrapulmonary cancer mortality (HR $1.43 ; 95 \% \mathrm{Cl} 1.06$ to 1.94$)$. The excess risk was mostly driven by patients with moderate and severe COPD. There was a trend towards a lower risk of cancer mortality among patients with COPD who used statins compared with patients with COPD who did not use statins (HR $0.57 ; 95 \% \mathrm{Cl} 0.32$ to 1.01). Interestingly, the risk of extrapulmonary cancer mortality was lower among statin users with COPD (HR 0.49; 95\% Cl 0.24 to 0.99).

Conclusions: COPD was associated with increased lung and extrapulmonary cancer mortality in this large cohort of patients with peripheral arterial disease undergoing vascular surgery. The risk of lung cancer mortality increased with progression of COPD. Statins were associated with a reduced risk of extrapulmonary cancer mortality in patients with COPD.

Chronic obstructive pulmonary disease (COPD) is a worldwide epidemic, affecting $10-15 \%$ of adults aged $\geqslant 40$ years. ${ }^{1}$ Over $80 \%$ of these patients have mild to moderately severe COPD (with forced expiratory volume in $1 \mathrm{~s}\left(\mathrm{FEV}_{1}\right)>50 \%$ predicted) and, in these patients, the leading cause of mortality is cancer which accounts for $>50 \%$ of all deaths. ${ }^{2}$ Previous studies have shown that, while smoking is a shared risk factor for both COPD and lung carcinoma, ${ }^{3}$ reduced lung function, independent of smoking, is another important risk factor for this type of cancer. ${ }^{4-10}$ However, several important questions remain unanswered. First, previous studies in this area have focused primarily on the incidence of lung cancer rather than on lung cancer mortality per se. Since early stage lung cancers are potentially curable and since patients with COPD may have more radiographic investigations, ascertainment bias may have confounded the previous studies that have used lung cancer incidence rather than mortality as the primary outcome. Second, although it is known that extrapulmonary cancers account for approximately $20 \%$ of all deaths in stage 1 and stage 2 COPD, ${ }^{11}$ the exact relationship between COPD (and its severity) and these cancers is not well known. Third, and most important from a clinical perspective, it is not known whether the risk of COPD and cancer mortality can be modulated by pharmacological treatment. Several studies suggest that statins (HMG-CoA reductase inhibitors) may reduce the risk of cancer including those in the lungs, pancreas, prostate and colon. ${ }^{12-15}$ On the other hand, some have suggested that statins may promote the development of new malignancies. ${ }^{16}$ However, none of these studies has evaluated the effects of statins on cancer mortality in patients with COPD. In the present study we sought to determine (1) the relationship between COPD (and its severity) and the risk of total cancer mortality and (2) whether the use of statins modified this relationship.

\section{METHODS}

\section{Study population}

The patient population for this study has been described previously. ${ }^{17}$ We used a cohort of 3371 patients who underwent elective non-cardiac vascular surgery (abdominal aortic surgery, carotid endarterectomy or lower limb arterial reconstruction) between 1990 and 2006. COPD was defined according to the guidelines of the Global Initiative for Chronic Obstructive Lung Disease (GOLD). ${ }^{18}$ The COPD diagnosis was based on a postbronchodilator $\mathrm{FEV}_{1}$ to vital capacity ratio of $70 \%$ or less on spirometry (which was the case in $82 \%$ of the patients with COPD). Patients without spirometry were classified based on the presence of pulmonary symptoms (dyspnoea, sputum production or cough) and use of COPD medications based on the GOLD Guidelines for Management of stable COPD patients. ${ }^{18}$ Mild COPD was defined as those who had symptoms and were using a short-acting bronchodilator when needed. Moderate COPD was defined as those with symptoms who required regular use of one or more bronchodilators. Severe COPD was defined as those with symptoms who were on regular treatment with one or more bronchodilators plus inhaled corticosteroids (for repeated exacerbations or persistent symptoms) or those patients who required domiciliary long-term oxygen therapy.

\section{Follow-up and end points}

At the time of follow-up the municipal civil registries were contacted to establish the survival status of all patients, which was completed in $96 \%$ 
of the patients during a median follow-up of 5 years (interquartile range (IOR) 2.0-9.1). The cause of death was ascertained by reviewing medical records, autopsy reports, contacting the referring physician, general practitioner or Statistics Netherlands. The primary end point of the study was total cancer mortality.

\section{Statistical analysis}

Cancer mortality rates among the COPD severity groups were compared using a $\chi^{2}$ test. Cumulative long-term survival was determined by the Kaplan-Meier method and compared using a log-rank test. Univariate and multivariate Cox regression analyses were used to determine the association between COPD (severity) and cancer mortality. In these analyses, patients without COPD were used as the reference group. Cox regression analysis was also used to determine the relationship between statins and cancer mortality. In the multivariate analysis we adjusted for age, gender, type of surgery, diabetes, smoking status, hypercholesterolaemia, corticosteroids, aspirin, statins and propensity score (for the association between statins and cancer mortality). The proportional hazards assumption was verified by visual inspection of the log-log survival curves and by using the Schoefeld residuals. This propensity score was developed using a multivariate logistic regression model to adjust for the likelihood of receiving statins and included age, gender, COPD severity, hypertension, hypercholesterolaemia, diabetes mellitus, renal dysfunction, current smoking status, obesity, type of surgery, year of surgery, cardiovascular history (table 1), pulmonary medications (bronchodilators and corticosteroids) and cardiac medications (beta-blockers and aspirin).

\section{RESULTS}

\section{Characteristics of study cohort}

The baseline characteristics of the cohort are presented in table 1 . The mean (SD) age was $66(12)$ years and $73 \%$ were men. There were 1310 patients (39\%) with COPD; mild COPD $(\mathrm{n}=578 ; 17 \%)$, moderate $\operatorname{COPD}(\mathrm{n}=579 ; 17 \%)$ and severe COPD $(n=153 ; 5 \%)$. Of the current smokers, $26 \%$ used statins whereas $23 \%$ of the never/ex-smokers were statin users $(p=0.20)$.

\section{COPD and cancer mortality}

Over the follow-up period, 316 patients (9\%) died from cancer. Patients with COPD had an increased risk of cancer mortality which was dependent on the severity of COPD. The risk of cancer mortality in patients with no COPD, mild, moderate and severe COPD was $8 \%, 10 \%, 14 \%$ and $12 \%$, respectively $(p<0.001)$. Overall, 102 patients $(3 \%)$ died from lung cancer. The risk of lung cancer mortality in patients with no, mild, moderate and severe COPD was $2 \%, 3 \%, 5 \%$ and $6 \%$, respectively $(p<0.001)$.

The cancer-free survival curves stratified by COPD severity are shown in fig 1 ( $p<0.001$, log-rank test) (see fig 1 in online supplement for lung cancer-free survival curves). COPD was independently associated with total cancer mortality (HR 1.61; 95\% CI 1.25 to 2.06) (table 2).

We tested the robustness of our findings by repeating the analysis following the exclusion of COPD patients without spirometry (see tables 1 and 2 in online supplement). It was reassuring that these data were very similar to those from the main analyses. In addition, in a secondary analysis we created a new category of patients with a restrictive disorder (defined as
Table 1 Patient characteristics

\begin{tabular}{|c|c|c|}
\hline & $\begin{array}{l}\text { Total } \\
(\mathrm{n}=3371)\end{array}$ & $\begin{array}{l}\text { Lung cancer } \\
(\mathrm{n}=102)\end{array}$ \\
\hline \multicolumn{3}{|l|}{ Demographics (\%) } \\
\hline Mean (SD) age (years) & $66(12)$ & $68(7)$ \\
\hline Men & 73 & 76 \\
\hline \multicolumn{3}{|l|}{ Type of surgery } \\
\hline AAA & 36 & 41 \\
\hline CEA & 24 & 18 \\
\hline LLR & 40 & 41 \\
\hline \multicolumn{3}{|l|}{ COPD (\%) } \\
\hline Mild & 17 & 19 \\
\hline Moderate & 17 & 29 \\
\hline Severe & 5 & 9 \\
\hline \multicolumn{3}{|l|}{ Cardiovascular history (\%) } \\
\hline Myocardial infarction & 22 & 17 \\
\hline Coronary revascularisation* & 16 & 14 \\
\hline Heart failure & 5 & 3 \\
\hline Angina pectoris & 14 & 8 \\
\hline Stroke or TIA & 30 & 24 \\
\hline \multicolumn{3}{|l|}{ Clinical characteristics (\%) } \\
\hline Diabetes mellitus & 15 & 10 \\
\hline Hypercholesterolaemia & 18 & 14 \\
\hline \multicolumn{3}{|l|}{ Smoking status } \\
\hline Never/ex-smoking & 72 & 64 \\
\hline Current smoking & 28 & 36 \\
\hline \multicolumn{3}{|l|}{ Medication } \\
\hline Corticosteroids & 10 & 15 \\
\hline Statins & 24 & 16 \\
\hline Aspirin & 40 & 31 \\
\hline
\end{tabular}

AAA, abdominal aortic surgery; CEA, carotid endarterectomy; COPD, chronic obstructive pulmonary disease; LLR, lower limb arterial reconstruction; TIA, transient ischaemic attack.

* Coronary artery bypass graft or percutaneous coronary intervention.

$\mathrm{FEV}_{1} / \mathrm{FVC}$ ratio $>80 \%$ with $\mathrm{FEV}_{1}<80 \%$ of predicted) and repeated the analysis. Patients with a "restrictive" defect had an increased risk of total cancer mortality as well as lung and extrapulmonary cancer mortality (see table 3 in online supplement).

A non-significant trend was observed for mild COPD (HR 1.30; $95 \%$ CI 0.95 to 1.79), whereas patients with moderate or severe COPD had a significantly increased risk of cancer mortality (HR 1.92; 95\% CI 1.43 to 2.58 and HR 1.95; 95\% CI 1.14 to 3.31, respectively). A similar association with even higher HR was found between COPD and lung cancer mortality (HR 2.06; 95\% CI 1.32 to 3.20). This association was largely driven by the group of patients with moderate and severe COPD, which were both strongly related to lung cancer mortality (HR 2.51; 95\% CI 1.50 to 4.17 and HR 3.38; 95\% CI 1.51 to 7.55 , respectively).

A sensitivity analysis was performed in which patients who died of lung cancer were excluded from the analysis. COPD was still associated with an increased risk for non-pulmonary cancer mortality (HR 1.43; 95\% CI 1.06 to 1.94). For patients with mild COPD the relationship was not significant (HR 1.22; 95\% CI 0.83 to 1.79 ), but the relationship with moderate COPD was significant (HR 1.70; 95\% CI 1.19 to 2.44). A significant association was not observed for severe COPD (HR 1.38; 95\% CI 0.67 to 2.86 ), probably due to competing risks for mortality.

\section{Statins and cancer mortality in all patients}

At baseline, 810 patients (24\%) received statin therapy. Of the patients who received this treatment, $6 \%$ died from cancer 


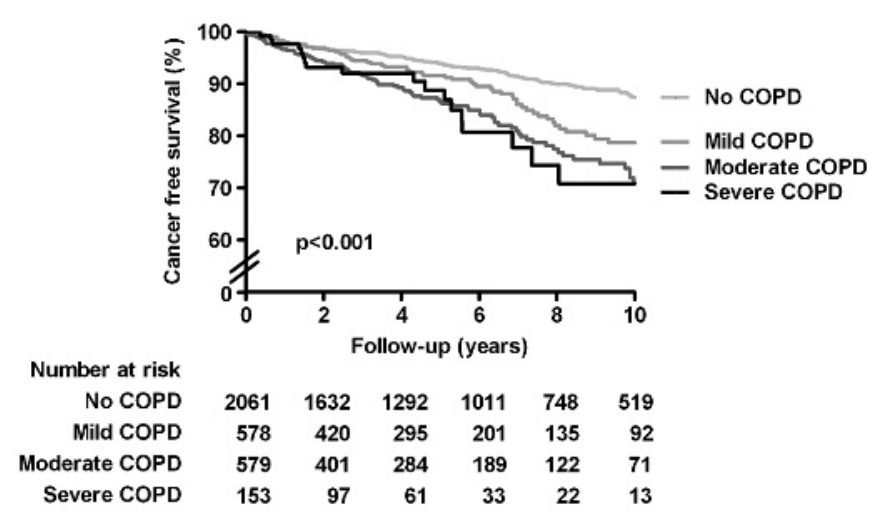

Figure 1 Long-term cancer-free survival according to severity of chronic obstructive pulmonary disease (COPD).

during the follow-up period compared with $11 \%$ of the patients who did not use statins $(\mathrm{p}<0.001) ; 2 \%$ of the patients who used statins died from lung cancer compared with $3 \%$ of those who did not use statins $(p<0.05)$. After excluding patients with lung cancer, extrapulmonary cancer was the cause of death in $4 \%$ of those who used statins and $7 \%$ of those who did not $(p<0.01)$. After adjustments for potential confounding factors, statins were not significantly associated with total cancer mortality (HR 0.82; 95\% CI 0.57 to 1.20), lung cancer mortality (HR 1.00; $95 \%$ CI 0.52 to 1.92 ) or extrapulmonary mortality (HR 0.74 ; $95 \%$ CI 0.47 to 1.17 ) (table 3 ).

\section{Statins and cancer mortality in patients with COPD}

Of the 1310 patients with COPD, 330 (25\%) used statins at baseline. More patients who did not use statins died from cancer than those who used statins $(14 \%$ vs $6 \%$; $<0.001)$. Similar differences were noted when only lung cancer mortality ( $5 \%$ vs $2 \%$; $<0.01$ ) or extrapulmonary cancer mortality ( $9 \%$ vs $4 \% ; \mathrm{p}<0.01)$ was considered. Figure 2 presents the long-term cancer-free survival curves of patients with COPD who did and did not use statins (see fig 2 in online supplement for lung cancer-free survival curves). Of the patients who used a statin, $87 \%$ survived during the follow-up period compared with $72 \%$ of those who did not use statins at baseline $(\mathrm{p}<0.01$, log rank test). After adjustments, the relationship was attenuated with borderline significance between statins and total cancer mortality (HR 0.57 ; 95\% CI 0.32 to 1.01 ) and no significance between statins and lung cancer mortality (HR 0.75; 95\% CI 0.28 to 2.05 ) (table 3 ). However, in this subanalysis including only patients with COPD, statins were associated with reduced cancer mortality from sites other than the lungs (HR 0.49; 95\% CI 0.24 to 0.99$)$.

\section{DISCUSSION}

This large study of patients with peripheral arterial disease demonstrates a significant relationship between COPD and cancer mortality. The risk rises sharply in patients with moderate to severe airflow obstruction $\left(\mathrm{FEV}_{1}<80 \%\right.$ predicted). Interestingly, both lung and extrapulmonary cancer deaths increased in patients with moderate COPD while, in those with severe COPD, lung cancer mortality predominated.

Our results confirm the findings of previous studies which investigated the association between COPD and lung cancer mortality. A severity-dependent association was found between the $\mathrm{FEV}_{1}$ quintiles and the risk for lung cancer mortality. ${ }^{19-22} \mathrm{~A}$ similar relationship was observed in our study; however, for the assessment of COPD severity we used the classifications as defined by the GOLD guidelines instead of $\mathrm{FEV}_{1}$ quintiles. We found in particular that moderate and severe COPD were independent risk factors for lung cancer mortality. These findings are also in line with the results of three other studies which also investigated the relationship between the GOLD classifications of COPD severity and lung cancer. However, our results could not be compared directly with these studies as they examined the association with lung cancer incidence rather than lung cancer mortality. ${ }^{923}$ Although the relationship between COPD and lung cancer is well known, ${ }^{19-22}$ the association of COPD with extrapulmonary cancers is less well established. A study by Purdue et al of construction workers failed to demonstrate a significant association between COPD and the risk of non-pulmonary carcinomas. ${ }^{7}$ However, the study was limited by the small number of cancer events $(3.7 \%)$ and the overwhelming predominance of men ( $96 \%$ of the cohort). By evaluating a group of high-risk patients (ie, older, smoking history, co-morbidities, mix of men and women), our study had sufficient power to demonstrate an independent association of COPD with extrapulmonary cancer mortality. On average, COPD increased extrapulmonary cancer mortality by more than $40 \%$. Our study also suggests that extrapulmonary cancers are particularly important causes of death in patients with moderate (but not mild or severe) COPD. ${ }^{19-21} 24$

These data are consistent with previous reports by the Lung Health Study (LHS) ${ }^{11}$ and TORCH (Towards a Revolution in COPD Health) groups. ${ }^{25}$ The LHS evaluated patients with mild to moderate COPD (average $\mathrm{FEV}_{1} 75 \%$ of predicted) whereas the TORCH trial studied patients with more severe diseases $\left(\mathrm{FEV}_{1} 44 \%\right.$ of predicted). In the LHS $21 \%$ of deaths were from extrapulmonary cancers while, in the TORCH trial, extrapulmonary cancers only accounted for $7 \%$ of the total deaths. Thus, in severe COPD, other competing causes of mortality such as lung cancer and respiratory failure may be important.

The mechanism by which COPD increases cancer risk is largely unknown. Cigarette smoking is a shared risk factor for

Table 2 Association between chronic obstructive pulmonary disease (COPD) and long-term cancer mortality

\begin{tabular}{|c|c|c|c|c|c|c|}
\hline & \multicolumn{2}{|c|}{ Total cancer mortality } & \multicolumn{2}{|c|}{ Lung cancer mortality } & \multicolumn{2}{|c|}{ Extrapulmonary cancer mortality } \\
\hline & Univariate & Multivariate* $^{*}$ & Univariate & Multivariate* & Univariate & Multivariate $^{*}$ \\
\hline & HR $(95 \% \mathrm{CI})$ & HR $(95 \% \mathrm{CI})$ & HR $(95 \% \mathrm{CI})$ & HR (95\% CI) & HR $(95 \% \mathrm{CI})$ & HR $(95 \% \mathrm{CI})$ \\
\hline COPD & 2.03 (1.63 to 2.53$)$ & $1.61(1.25$ to 2.06$)$ & $2.76(1.86$ to 4.08$)$ & $2.06(1.32$ to 3.20$)$ & $1.76(1.34$ to 2.30$)$ & $1.43(1.06$ to 1.94$)$ \\
\hline No COPD & 1.00 & 1.00 & 1.00 & 1.00 & 1.00 & 1.00 \\
\hline Mild COPD & $1.62(1.20$ to 2.18$)$ & $1.30(0.95$ to 1.79$)$ & $1.94(1.13$ to 3.32$)$ & 1.52 (0.87 to 2.68$)$ & $1.49(1.04$ to 2.15$)$ & $1.22(0.83$ to 1.79$)$ \\
\hline Moderate COPD & $2.39(1.83$ to 3.12$)$ & 1.92 (1.43 to 2.58$)$ & $3.25(2.04$ to 5.17$)$ & $2.51(1.50$ to 4.17$)$ & $2.06(1.48$ to 2.87$)$ & $1.70(1.19$ to 2.44$)$ \\
\hline Severe COPD & $2.42(1.49$ to 3.95$)$ & $1.95(1.14$ to 3.31$)$ & $4.54(2.21$ to 9.34$)$ & $3.38(1.51$ to 7.55$)$ & $1.64(0.83$ to 3.25$)$ & $1.38(0.67$ to 2.86$)$ \\
\hline
\end{tabular}


Table 3 Association between statins and cancer mortality

\begin{tabular}{|c|c|c|c|c|c|c|}
\hline & \multicolumn{2}{|l|}{ Cancer mortality } & \multicolumn{2}{|c|}{ Lung cancer mortality } & \multicolumn{2}{|c|}{ Extrapulmonary cancer mortality } \\
\hline & Univariate & Multivariate* & Univariate & Multivariate* & Univariate & Multivariate* \\
\hline & HR (95\% CI) & HR $(95 \% \mathrm{CI})$ & HR $(95 \% \mathrm{CI})$ & HR $(95 \% \mathrm{CI})$ & HR $(95 \% \mathrm{CI})$ & HR (95\% CI) \\
\hline \multicolumn{7}{|c|}{ All patients } \\
\hline Statins & $0.70(0.52$ to 0.96$)$ & $0.82(0.57$ to 1.20$)$ & $0.74(0.43$ to 1.27$)$ & $1.00(0.52$ to 1.92$)$ & $0.69(0.47$ to 1.00$)$ & $0.74(0.47$ to 1.17$)$ \\
\hline \multicolumn{7}{|c|}{ Patients with COPD } \\
\hline Statins & $0.52(0.32$ to 0.84$)$ & $0.57(0.32$ to 1.01$)$ & $0.46(0.20$ to 1.07$)$ & $0.75(0.28$ to 2.05$)$ & $0.56(0.31$ to 1.00$)$ & $0.49(0.24$ to 0.99$)$ \\
\hline
\end{tabular}

${ }^{*}$ Adjusted for age, gender, type of surgery, diabetes, smoking, hypercholestrolaemia, corticosteroids, aspirin and propensity score.

both COPD and solid organ cancers including lung, breast and colorectal cancer. However, in our analysis we adjusted for smoking and still found a significant relationship between COPD and cancer. Moreover, a recent study of lifelong never smokers found a significant relationship between COPD and lung cancer mortality. ${ }^{8}$ Collectively, these data suggest that, in addition to cigarette smoking, other potential explanations should be considered. These include (1) shared genetic susceptibility; ${ }^{26}{ }^{27}$ (2) delayed clearance of inhaled carcinogens because of airflow limitation; ${ }^{28}$ and (3) chronic low-grade lung and systemic inflammation associated with COPD. ${ }^{29} \mathrm{We}$ have found previously that, in patients with COPD, systemic levels of Creactive protein, a biomarker of systemic inflammation, is associated with a future risk of cancer mortality including those related to extrapulmonary cancers. ${ }^{30}$ Similarly, in the largest meta-analysis of its kind, Danesh and colleagues showed that plasma fibrinogen, another non-specific marker of systemic inflammation, is associated with both pulmonary and extrapulmonary cancers in smokers and never smokers. ${ }^{31}$

Statins are lipid-lowering drugs that also possess significant anti-inflammatory properties which may have beneficial effects not only on cardiovascular disease but on other co-morbidities associated with COPD. The findings from the present study suggest that, in patients with COPD, statins may be effective in reducing deaths, especially from extrapulmonary cancers. The results of our study are consistent with previous epidemiological studies which have suggested that statins may lower cancer risk. ${ }^{12-15} 32{ }^{33}$ A study by Blais and collegues ${ }^{34}$ found that patients using statins were less likely to be diagnosed with cancer than those using bile acid-binding resins, which indicates that the protective effect of statins is unlikely to be due to their lipidlowering effect but is probably the result of other effects. The increased risk of cancer conferred by COPD might explain the increased benefit of statins observed in patients with COPD compared with patients with normal pulmonary function. Randomised controlled trials are needed to confirm our findings.

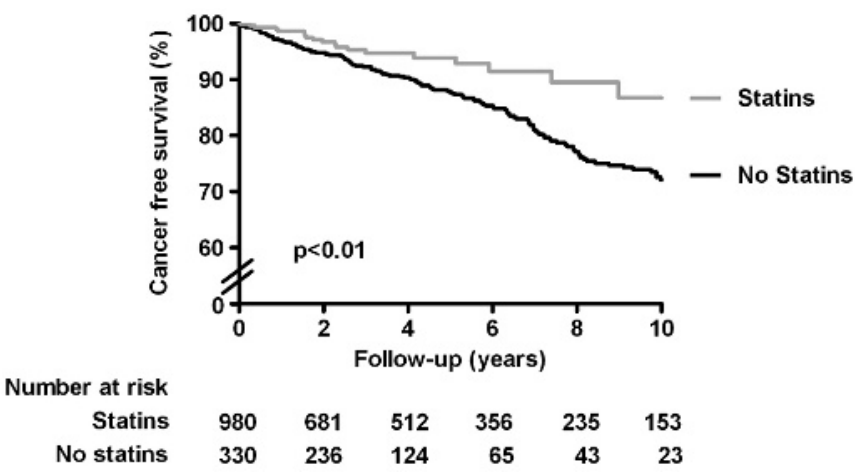

Figure 2 Long-term cancer-free survival according to statin use in patients with chronic obstructive pulmonary disease.
There are several limitations to this study. First, as the data were collected retrospectively, residual confounding by measured and unmeasured variables is possible. The results of this study should thus be interpreted cautiously and conservatively. Second, the study population consisted of patients who underwent vascular surgery. Since such patients are more likely to be smokers and to have additional risk factors for cancer than those of the general population, the findings of the present study may not be generalisable to the total population. Third, we did not have complete data on the primary site of the extrapulmonary cancer deaths. Of the 214 patients who died from extrapulmonary cancers, we were able to specify the site in 124 (58\%) patients (see fig 1 in online supplement for details). The impact of statins on different types and sources of extrapulmonary cancers therefore remains unknown. Fourth, statin use was categorised based on whether or not patients were treated with statins at the start of the follow-up period. We did not consider change in statin status during the followup period. This may have caused misclassification of statin status, which would have diluted the relationship. Our estimate of the potential effect of statins on cancer mortality is therefore likely to be conservative.

In conclusion, COPD was associated with increased lung and extrapulmonary cancer mortality in this large cohort of patients with peripheral arterial disease undergoing vascular surgery. The risk of lung cancer mortality increased with severity of COPD, whereas the risk of extrapulmonary cancer was significant only in patients with moderate COPD, possibly because of competing risks for mortality in patients with severe COPD. Statins may modulate the risk of extrapulmonary cancer mortality in patients with COPD.

Contributors: All authors contributed to the study conception and design, revision of the manuscript and approved the final version. In addition, YRBMvG, SEH, VH, HS and RTVD were responsible for the statistical analysis and YRBMvG, SEH, DDS, JJB and DP contributed to the interpretation of the analysis. YRBMvG, DDS and DP were responsible for writing of the manuscript. VH, HS, FWM and RTVD took part in the data acquisition and DP supervised the study and was responsible for the funding source.

Funding: YRBMvG and SEH are supported by an unrestricted research grant from "Lijf and Leven" Foundation, Rotterdam, The Netherlands.

Competing interests: None.

Ethics approval: The Medical Ethics Committee of the hospital was informed about the study and no official approval was requested per institutional practice.

Provenance and peer review: Not commissioned; externally peer reviewed.

\section{REFERENCES}

1. Buist AS, McBurnie MA, Vollmer WM, et al. International variation in the prevalence of COPD (the BOLD Study): a population-based prevalence study. Lancet 2007;370:741-50.

2. Anthonisen NR, Connett JE, Kiley JP, et al. Effects of smoking intervention and the use of an inhaled anticholinergic bronchodilator on the rate of decline of FEV ${ }_{1}$. The Lung Health Study. JAMA 1994;272:1497-505.

3. Herbst RS, Heymach JV, Lippman SM. Lung cancer. N Eng/ J Med 2008;359:1367-80.

4. Wasswa-Kintu S, Gan WO, Man SF, et al. Relationship between reduced forced expiratory volume in one second and the risk of lung cancer: a systematic review and meta-analysis. Thorax 2005;60:570-5. 
5. Frostad A, Soyseth V, Haldorsen T, et al. Impact of respiratory symptoms on lung cancer: 30-year follow-up of an urban population. Lung Cancer 2008;60:22-30.

6. Kishi K, Gurney JW, Schroeder DR, et al. The correlation of emphysema or airway obstruction with the risk of lung cancer: a matched case-controlled study. Eur Respir J 2002;19:1093-8.

7. Purdue MP, Gold L, Jarvholm B, et al. Impaired lung function and lung cancer incidence in a cohort of Swedish construction workers. Thorax 2007;62:51-6.

8. Turner MC, Chen Y, Krewski D, et al. Chronic obstructive pulmonary disease is associated with lung cancer mortality in a prospective study of never smokers. Am J Respir Crit Care Med 2007;176:285-90.

9. Wilson DO, Weissfeld JL, Balkan A, et al. Association of radiographic emphysema and airflow obstruction with lung cancer. Am J Respir Crit Care Med 2008;178:738-44.

10. de Torres JP, Bastarrika G, Wisnivesky JP, et al. Assessing the relationship between lung cancer risk and emphysema detected on low-dose CT of the chest. Chest 2007;132:1932-8.

11. Anthonisen NR, Connett JE, Enright PL, et al. Hospitalizations and mortality in the Lung Health Study. Am J Respir Crit Care Med 2002;166:333-9.

12. Farwell WR, Scranton RE, Lawler EV, et al. The association between statins and cancer incidence in a veterans population. J Natl Cancer Inst 2008;100:134-9.

13. Khurana V, Bejjanki HR, Caldito G, et al. Statins reduce the risk of lung cancer in humans: a large case-control study of US veterans. Chest 2007:131:1282-8.

14. Khurana V, Sheth A, Caldito G, et al. Statins reduce the risk of pancreatic cancer in humans: a case-control study of half a million veterans. Pancreas 2007;34:260-5.

15. Graaf MR, Beiderbeck $A B$, Egberts $A C$, et al. The risk of cancer in users of statins. $J$ Clin Oncol 2004;22:2388-94.

16. Shepherd J, Blauw GJ, Murphy MB, et al. Pravastatin in elderly individuals at risk of vascular disease (PROSPER): a randomised controlled trial. Lancet 2002;360:1623-30.

17. van Gestel YR, Hoeks SE, Sin DD, et al. Impact of cardioselective beta-blockers on mortality in patients with chronic obstructive pulmonary disease and atherosclerosis. Am J Respir Crit Care Med 2008;178:695-700.

18. Rabe KF, Hurd S, Anzueto A, et al. Global strategy for the diagnosis, management, and prevention of chronic obstructive pulmonary disease: GOLD executive summary. Am J Respir Crit Care Med 2007;176:532-55.

19. Eberly LE, Ockene J, Sherwin R, et al. Pulmonary function as a predictor of lung cancer mortality in continuing cigarette smokers and in quitters. Int $J$ Epidemiol 2003; 32:592-9.
20. Hole DJ, Watt GC, Davey-Smith G, et al. Impaired lung function and mortality risk in men and women: findings from the Renfrew and Paisley prospective population study. BMJ 1996;313:711-6.

21. Kuller LH, Ockene J, Meilahn E, et al. Relation of forced expiratory volume in one second $\left(\mathrm{FEV}_{1}\right)$ to lung cancer mortality in the Multiple Risk Factor Intervention Tria (MRFIT). Am J Epidemiol 1990;132:265-74.

22. Van den Eeden SK, Friedman GD. Forced expiratory volume (1 second) and lung cancer incidence and mortality. Epidemiology 1992;3:253-7.

23. Mannino DM, Aguayo SM, Petty TL, et al. Low lung function and incident lung cancer in the United States: data From the First National Health and Nutrition Examination Survey follow-up. Arch Intern Med 2003;163:1475-80.

24. van Eeden SF, Sin DD. Chronic obstructive pulmonary disease: a chronic systemic inflammatory disease. Respiration 2008;75:224-38.

25. McGarvey LP, John M, Anderson JA, et al. Ascertainment of cause-specific mortality in COPD: operations of the TORCH Clinical Endpoint Committee. Thorax 2007;62:411-5.

26. Schwartz AG, Ruckdeschel JC. Familial lung cancer: genetic susceptibility and relationship to chronic obstructive pulmonary disease. Am J Respir Crit Care Med 2006;173:16-22.

27. Young RP, Hopkins RJ, Hay BA, et al. Lung cancer gene associated with COPD: triple whammy or possible confounding effect? Eur Respir J 2008;32:1158-64.

28. Yanai M, Hatazawa J, Ojima F, et al. Deposition and clearance of inhaled 18FDG powder in patients with chronic obstructive pulmonary disease. Eur Respir $J$ 1998:11:1342-8.

29. Hogg JC. Pathophysiology of airflow limitation in chronic obstructive pulmonary disease. Lancet 2004;364:709-21.

30. Man SF, Connett JE, Anthonisen NR, et al. C-reactive protein and mortality in mild to moderate chronic obstructive pulmonary disease. Thorax 2006;61:849-53.

31. Danesh J, Lewington S, Thompson SG, et al. Plasma fibrinogen level and the risk of major cardiovascular diseases and nonvascular mortality: an individual participant meta-analysis. JAMA 2005;294:1799-809.

32. Cauley JA, McTiernan A, Rodabough RJ, et al. Statin use and breast cancer: prospective results from the Women's Health Initiative. J Natl Cancer Inst 2006;98:700-7.

33. Poynter JN, Gruber SB, Higgins PD, et al. Statins and the risk of colorectal cancer. N Engl J Med 2005;352:2184-92.

34. Blais L, Desgagne A, LeLorier J. 3-Hydroxy-3-methylglutaryl coenzyme A reductase inhibitors and the risk of cancer: a nested case-control study. Arch Intern Med 2000;160:2363-8. 